which could not be opened. Hydrops is a very rare condition. I thought it did not exist until Dr. Herman Knapp made his interesting report of some cases, the bistory of one of which was very much like this.

Dr. Max Thorner-There seems to be no doubt that the headaches were due to some intracranial pressure, though the ophthalmoscopic examination showed nothing unusual. In a case shown by Dr. Woakes in 1885 at the London Hospital for Diseases of the Throat and Chest, he considered the discharge from the nose to be cerebro-spinal fluid, in a case that bore close resemblance to the one reported A chemic and microscopic examination, if I remember rightly, was not made at the time. The discharge occurred intermittently and was always preceded by headache. A communication between the arachnoid space and the lymphatics of the nose has been proved to exist by Flatau's magnificent demonstrations, made at the International Medical Congress at Berlin, in 1890. This fact accounts for many otherwise obscure phenomena.

Dr. J. A. Stucky-From the history of the case and the quantity of the discharge, I am almost forced to conclude that the discharge was from the cranial cavity. There would not be over an ounce or so from the ethmoidal cells, even if they were much enlarged.

Dr. G. V. WOOLEN-I have often been surprised at the amount of discharge in the ethmoidal cells and at the large quantity of polypi that can be taken from this region. The amount of operative tolerance in this locality is remarkable at times. It does not seem that the fluid was cerebro-spinal, or there would have been other cerebral symptoms besides pain, such as coma, paralysis, etc.

Dr. A. DE VILBISS-Although many have done considerable work in the region of the roof of the nose, we should not invade that region without some fear of doing harm. Only about four months ago I witnessed the death of a patient from an operation in that direction. In old cases where we have empyema we can operate more readily than in acute cases. That the cerebro-spinal fluid can leak out and escape through the nasal cavity I believe, though I have never made any postmortem demonstration of the avenues by which it comes. I had a patient who, every few months, by bending the head forward could discharge about half a teaspoonf ul of cerebro-spinal fluid through the nose. He had also symptoms of focal epilepsy. He was relieved by puncturing the dura and drawing off the cerebro spinal fluid.

Dr. SCHEPPEGRELL-This case could not be one of hydrops of the ethmoidal cells, as this would not account for the sud den and complete discharge of the fluid, which indicates the existence of a kind of buffer, which in this region could be fur nished only by the intracranial pressure. As already stated, a cystic accumulation in the subarachnoid space would alone explain the character of the fluid examined and the various symptoms presented in this case.

\section{THE TREATMENT OF EMPYEMA OF THE FRONTAL SINUS.}

Discussion in the Section on Laryngology and Otology, at the Forty eighth Annual Meeting of the American Medical Association, held at Philadelphia, June 1-4, 1897.

OpEned By J. H. BRYAN, M.D.

$$
\text { WAshington, D. C. }
$$

In accepting your invitation to open the discussion on this most important and interesting subject, I feel that I can not do better than first to call your attention to a few of the anatomic peculiarities of the frontal sinuses, in order that the pathology and treatment of chronic suppurative inflammations of these cavities can be the better understood.

The size of the frontal sinuses varies greatly in different subjects, and frequently in the same subject the variation is very marked, one cavity occasionally being quite large while the other is many times smaller, or absent altogether.

After an examination of 120 skulls made by Herbert Tilley ${ }^{1}$ he considers a sinus normal when it measures $28 \mathrm{~mm}$. from the median line outward to about the junction of the inner and middle third of the supraorbital ridge; and in vertical extent measured from the root of the nose from 20 to $22 \mathrm{~mm}$. Lamb, ${ }^{2}$ who has also investigated the size of these cavities, found the right one with a varying capacity from one and one-third to six c.c. while that of the left sinus was from one and one-fourth to four and two-thirds c.c. As showing the difference existing between the two sides he found in one instance the left sinus was to the right as seventy to ninety-five.

In health these cavities are separated by a very thin partition or septum which in the majority of instances is complete, although they occasionally communicate through a small opening. The septum is occasionally not straight, deviating to one side or the other thereby rendering one cavity smaller than the other.

Situated between the frontal and ethmoidal sinuses there is a series of cells formed by the junction of the ethmoid and frontal bones, but which form no part of either of these cavities. They, however, bear a very important relation to both sinuses, for when affected by an extension of a morbid process in either of the adjoining cavities they seriously complicate these conditions and render the prognosis much more uncertain. I believe in nearly all severe cases of empyema of either the frontal or ethmoidal cavities these cells are to a greater or less degree, affected.

Chronic empyema of the frontal sinuses while not an uncommon occurrence in some of the continental countries, as Russia and Germany, has not been so frequently observed in this country until within the past few years, and especially since the frequent visitations of epidemic influenza, which seems to have a special predilection for the accessory sinuses. While chronic suppuration of this cavity may and does frequently arise from other causes such as an extension of simple catarrhal inflammations from the nasal cavities, and from the retention of the secretions in the sinus by obstructive lesions within the nose, the most frequent cause in my experience has been the extension of the morbid process from the nose in the course of an attack of grippe.

While the prognosis of this affection is generally favorable the treatment will be found to be difficult and tedious according to the methods adopted for its relief.

Since the rapid advances made in the study of diseases of the nose there is a tendency on the part of some rhinologists to treat this affection through the nose, especially since Jurasz has pointed out the feasibility in a few cases of passing a probe through the infundibular orifice in the nose. There is no doubt that in a few instances this can be done, but in the majority of cases it will be found to be impossible to pass a probe into the frontal sinus through the frontonasal duct, for generally this duct is situated far back, and is occasionally so tortuous that no probe can be made to follow its course. When sounding the frontal sinus is possible treatment conducted through the nose without first making an external opening is only suitable for acute cases.

Max Schaeffer has proposed opening the sinus by making a forced opening through the nose. It is only necessary to mention this method to condemn it as very dangerous, for there is already one fatal case, by this method, on record. Any one who has paid any attention to the anatomic irregularities that exist in this region can not feel assured that he can enter the sinus by forcing an instrument through the middle meatus or middle turbinated body. Even should one be successful in entering the sinus by either of the above mentioned methods little could be 
accomplished in relieving the inflammatiom, for in these chronic cases the pus is very thick, best described as stringy in character; the cavity is generally filled with granulation or polypoid tissue, and there is frequently some caries of the surrounding bony walls. When such conditions exist no amount of irrigation with antiseptic lotions will relieve the inflammation. I can therefore speak of these intranasal methods only to advise against their employment in chronic frontal sinusitis of long standing. I think most authorities of any extended experience will agree that one of the external methods will in the majority of cases be called for, and the earlier the operation is done the shorter will the usual subsequent treatment be.

There are several methods proposed for entering the frontal sinus. One, which I employed in a case successfully, but in which the subsequent treatment was very tedious, is where the incision is made along the lower border of the supra-orbital ridge, first pulling the integument forcibly up on the forehead so that the line of incision would fall just under the eye-brow. The incision beginning at the root of the nose and extending outward from an inch to an inch and a half is made down to the bone, the integument and periosteum elevated, and the sinus opened at the internal angle either by means of a drill, chisel or trephine. This opening does not admit of a thorough inspection of the interior of the cavity, and any caries of the septum or fronto-ethmoidal cells could easily escape detection. The disadvantages of this method are that the opening does not admit enlargement of the frontonasal duct as readily as that through the anterior wall, consequently the cavity has to be irrigated through a rubber or metallic drainage tube introduced into the sinus at this point, which results in the patient recovering with a decided depression over the opening into the sinus, and occasionally with a fistulous opening remaining.

Panas, ${ }^{3}$ who has had a large experience in treating this affection, recommends that an incision be made along the lower border of the supra-orbital ridge and then makes another perpendicular to this upon the base of the nose, the triangular flap including integument and periosteum thus formed is detached from the bone, and an opening made into the sinus near the inner angle of the orbit large enough to admit of a thorough exploration of the cavity.

Another method, which offers many advantages over the above mentioned procedures, is that devised by Ogston * of Aberdeen, in 1884, and more recently and independently advocated by Luc. ${ }^{5}$ In this operation the incision is made in the median line commencing at the root of the nose and extending from an inch and a half to two inches on the forehead. The skin and periosteum are elevated and a centimeter of bone removed from the anterior wall of the sinus by means of a crown trephine applied several lines from the median line and about two or three lines above the supra-orbital ridge. By this means an opening of sufficient size is made so as to admit of a thorough exploration of the sinus, and caries of the bony walls, the septum, or of the fronto-ethmoidal cells can be easily detected and remover. After the removal of any polypoid or necrotic tissue the cavity is thoroughly cleansed, and the fronto-nasal duct enlarged by passing a trocar from the sinus into the nose, using the little finger as a guide, after which a self-retaining drainage tube is introduced through this enlarged opening and allowed to remain as long as necessary. The membrane lining the cavity may be touched with a 20 per cent. solution of the chlorid of zinc and the wound closed by means of an interrupted or a subcutaneous suture, which should then be hermetically sealed with a solution of iodoform and collodion. The subsequent irrigations can be made through the drainage tube until all secretions have ceused to form.

The advantages of this method of treating chronic abscesses of the frontal sinus are manifest in that the cavity with all of its irregularities is rendered thoroughly accessible, and all diseased tissue can be removed before closing the wound. There is no depression left after the removal of the button of bone from the anterior wall of the sinus, and only a slight scar remains which in carefully managed cases amounts to little more than an exaggeration of the natural cleav. age of the skin so frequently observed in this region.

The duration of treatment is very materially reduced by this method. In a case I reported at the recent meeting of the American Laryngological Association the duration of treatment was a little over six weeks from the date of the operation, and it was somewhat prolonged in this case owing to an unfortunate accident which resulted in suppuration taking place in the line of incision, either from an imperfectly sterilized cat-gut suture, or an infection of the under surface of the wound from the cavity below.

Bote ${ }^{6}$ has modified this operation somewhat with the view of preventing the scar that may be left after the median operation. After shaving the inner twothirds of both eyebrows, he makes an incision the length of the internal third of the supra-orbital ridge, which is prolonged to the root of the nose. After elevating the integument and periosteum he removes the external plate of the sinus near the root of the nose, and then carefully detaches fragments of the anterior ethmoid cells until a large communication is left between the frontal and nasal cavities; the sinus is cleared of all diseased tissue and a strip of iodoform gauze introduced through the opening into the nose, when the external wound is closed.

This operation would seem to present some advantages over that of the Ogston-Luc method, especially in not leaving much of scar, and also in having the fronto-nasal opening sufficiently large to do away with a drainage tube.

After the artificial opening has been made the sub. sequent treatment consisis in keeping the cavity free from all secretions, which is done by frequent irrigations with antiseptic lotions. The use of strong antiseptics in this cavity as well as in the other accessory sinuses can not be too strongly condemned. The best results are obtained after the persistent use of such mild applications as hydrogen dioxid and a saturated boric acid solution.

Dr. Max Thorner-Diseases of the frontal sinus, acute as well as chronic, are often of such a nature that diagnosis is easy, but in some cases it is very difficult. It is often extremely hard to find from which of the accessory cavities the discharge takes place. A patient who was brought to my service in the Cincinnati Hospital had previously all the accessory cavities of one side opened without finding the

1 Lancet, Iondon, Sept. 26, 1896.

Reference Handbook iredical Science, Vol, vii. p. 659

Archives of Oph thalmology. Vol. xx. p. 658

Medical Chronicle. Dec. 188

Archiv. intermat. de laryngol. Par. 1846, ix. 163.17s.

ev. hehdomadaire de laryngolowie, d'otologie et de thinologie, No. 3 Jan. 16,1897 . 
cause of persistent headache. It had been rather the the difficulty of entering the frontal sinus through the habit of late to operate on the accessory cavities in nose, I have done it a great many times, and have been headache which can not be traced to any other cause. sure that the probe reached the cavity. If the case It is almost impossible to gain admission into the has not gone to the point of caries, we should try the frontal sinus through the nose, although I will not intranasal treatment first.

deny that it can occasionally be done. Some time ago I tried, on the cadaver, to introduce probes through the nose into the frontal sinus, and was astonished to find in how many different directions my probe had been conducted. Some observers have repeatedly stated that it was very easy to introduce a probe or flexible canula, but I do not think so. Even if there is only one case on record where death has followed the attempt at opening the frontal sinus from the nose others have occurred. Whatever may be the opinion regarding acute, $I$ think that all agree that in chronic cases we should do the external operation. Operative treatment through the nose in such cases is almost impossible, and always dangerous.

The method mentioned by Dr. Bryan is among those almost universally used. Of late I have changed my method slightly. I do not make the incision in a straight line along the eyebrow, but following the eyebrow about two-thirds its length, and then curve it slightly upward toward the median line. I have found that access to the cavity is better, while the scar is almost invisible. In chronic cases, where there is extensive caries it is almost impossible, in my experience, to get healing in four to six weeks. In the modern, or so-called radical operation upon the middle ear cavities, we have the advantage that we plaster the skin into the wound-cavity to cover the raw bone surfaces, but we can not do that in the frontal sinus, though I think it has been proposed. Healing takes almost twice as long where there is extensive caries, especially where the conditions for drainage are not favorable. I have found it most advantageous in such cases to drain for some time at least through the external opening although it takes longer. In all cases where I found extensive caries, I left a little hole for drainage, and the results were better than where I had closed the external wound by sutures at the time of the operation and depended upon drainage through the nose alone. This method does not preclude closing the external wound at once in cases where we find no caries at all, or only to a slight extent.

Dr. J. A. Stucky-I have seen quite a number of these cases, and in every one there has been serious trouble in the middle meatus, and the middle turbinal has been enlarged. I have found that removal of the anterior end of the middle turbinal gave great relief. I believe the orifice of the infundibulum is closed by adhesions, and if we succeed in opening the process and subduing the inflammatory conditions, nature will often complete the case. By frequent cleansing of the nasal cavity and keeping the patient quiet, especially in acute cases, we can prevent more serious attacks. As to chronic cases, $I$ am in favor of the external operation. I believe in making a larce opening to see what you are doing and also to get free drainage. I prefer to make the opening with the trephine, at the root of the nose. I do not like the opening at the eyebrow, which I have tried but have had much trouble and swelling.

Dr. ScheppegrelL-Where the external operation nal before operation and also the ethmoid bone which is necessary it should be done by the radicul method lies between the infundibulum and the upper part of which Dr. Bryan suggests. We should not however the septum.

proceed to external operation too soon. The attempt As to immediate closure of the external wound, the at intranasal treatment should be made first. As to new membrane forms just as well in the closed dark 
cavity as in the light. I have tried to ascertain how the bare bone closed over in these cavities. In one case I reopened, and found the granulations very exuberant and softened by the atmosphere which passed upward in respiration.

The Chatrman - We must draw a sharp line of distinction between acute and chronic cases. Our reports and statistics are vitiated by dragging in acute with chronic cases. The majority of acute cases recover spontaneously, and the operation receives the credit of the cure. Intranasal methods should be carried out tentatively, especially where there is polypoid degeneration of the middle turbinal or the presence of actual polypi. The nose should be cleared and the anterior end of the turbinal removed if necessary. The operation by the intranasal method often dnes not result in a perfect cure, though it often relieves the headache and may establish better drainage. Intranasal treatment should not be carried on too long; in this way we may weary and discourage our patients. After having done what is really essential by intranasal treatment we should proceed to the external operation. I consider Luc's a great advance over previous methods in the external treatment of this disease. It will enable us to promise a cure if there is no caries, and it leaves no deformity. This operation has been well described by Dr. Bryan, but there are several points that were not quite clear to me. First, as to whether the incision is made exactly in the median line or deviated a little to one side; second, in reference to the modification of Luc's operation, in which there is an incision along the orbital arch and then a vertical one connected with it, with a view to lessening the deformity.

Dr. BRYAN - I invariably relieve the intranasal conditions as far as possible before resorting to operation. The removal of the middle turbinated body is sometimes necessary when the ethmoidal cells are involved. These cases can not be cured unless every bit of caries and pus is removed. After the cavity has been thoroughly curetted and the diseased tissue removed, I know of no reason why the patient shonld have an open wound until the so-called healing of the internal cavity takes place; this occurs just as rapidly when the wound is closed. As to the incision, my last case had a decided furrow in the median line and $I$ followed this. It seems to me that Botey's method is a great advance over the median operation, though $\mathrm{I}$ have never tried it. The incision is described as commencing at the inner third and under surface of one eyebrow and then carried across the root of the nose, the integument and periosteum elevated, and the opening made just above the inner angle. The great advantage is that the rubber drainage tube is dispensed with. The communication between the sinus and the nose is a large one.

Gerson's Elastic Suspensory Bandage.-A soft elastic cloth, fringed on one edge, is covered with "York's rubber zinc plaster" and applied in epididymitis as follows: The scrotum is freed from hair and moisture and the bandage is applied around the empty scrotum below the testes, with the fringe up to prevent cutting. The scrotal skin thus drawn tight exerts constant pressure on the swollen testes and reduces the swelling. When the bandage becomes loose, as the swelling subsides, it is removed and applied higher up until complete recovery. In case of varicocele the bandage is removed at night. Frank and Lassar endorsed from personal experience the usefulness of this bandage.

\section{MENIERE'S DISEASE, WITH REPORT OF} A CASE.

Presented in the Section on Laryngology and Otology at the Forty. eighth Annual Meeting of the American Medical Association, held at Philadelphia, Pa., June 1-4, 1897. BY JAMES M. BROWN, M.D.

Instructor in Oto'ogy in the University of Pennsylvania; Assistant Aural Surgeon to the Hospital of the University of Pennsylvania; Aural and Ophthalmic Surgeon to the Western Temporary Home; Aurist to the Presbyterian Orphanage. AND JUDSON DALAND, M.D.

Instructor in Clinical Medicine and Lecturer on Physical Diagnosis in the Cuiversity of Pennsylvania; Assistant Physician to the Hospital of the University of Pennsylvania; Professor of Clin. ical Medicine in the Philadelphia Polyclinic. PHILADELPHIA, PA.

The employment of a term as indefinite as "Meniere's Disease" is now generally held to be, in giving title to the subject to which I ask your attention, may call for some explanation or apology, for quoting the words of Sir Wm. B. Dalby: "The term serves more frequently to mark ignorance of the lesion which occasions a series of symptoms, often analogous, but which are under the influence of very different causes." Such apology if called for must be the admitted absence of any designation more definite, and yet generally applicable to all cases of, or the various symptoms met with in, this disease, together with the great diversity of opinion which still obtains in the minds of the profession concerning it. Until we know the exact pathology and are able to separate other analogous diseases with which it may be confounded, it is wiser to hold to the name "Meniere's disease." Faintness, deafness and vertigo were the symptoms that especially engrossed Meniere's attention, and of these the latter seemed most momentous, and his investigations led him to believe that the lesions which produced it were restricted to the semicircular canals. That this is not the universally accepted opinion is proven by the widely divergent expressions of master minds engaged in the study of both its lesions and symptoms. Politzer and Lucae both report instances in which the semi-circular canals were absent or, by trauma, filled with blood, and yet the patients did not suffer from any disturbance of equilibrium. Gradenigo, Moos and Burnett believe the vertigo to be chiefly due to an extension to the labyrinth of a chronic catarrhal affection of the middle ear. Steiner of Cologne believes that the vertiginous symptoms result from lesions within the brain or its membranes, causing alterations in pressure. Baginsky, Randall, Dench and others regard Meniere's disease as a complex of symptoms, in which the cause can not be due to disease of the canals alone, but whether labyrinthine involvement, such as hemorrhage or serous effusion, is present in all cases is a question, as yet, not certainly answered. It may be caused by organic changes in the perceptive mechanism, or secondary to diseases of the conductive apparatus, or to blood conditions, or circulatory disturbances, to certain diatheses, or may be wholly reflex. Mettler says it is too complicated to be considered as a mere disarrangement of the function of the semicircular canals alone, and yet it may be considered that in true Meniere's disease these only are affected, and that aural vertigo may not be aural at all, except so far as having certain aural symptoms associated with the vertigo are concerned. Bezold has collected and reports forty-six cases of necrosis of the labyrinth, and notes that only twelve showed symptoms of vertigo. 